\title{
Studies on apparent free cortisol and testosterone in plasma from patients with breast tumours
}

\author{
M. K. JONES* \\ M.R.C.P. \\ G. I. DYER* \\ I. D. RAMSAY $\dagger$ \\ M.D., M.R.C.P., M.R.C.P.E. \\ W. P. Collins $\ddagger$ \\ Ph.D., D.Sc.
}

\begin{abstract}
*Faith Courtauld Unit for Human Studies in Cancer, $\ddagger$ Department of Obstetrics and Gynaecology, King's College Hospital Medical School, London, and †Regional Endocrine Centre, North Middlesex Hospital, London
\end{abstract}

\begin{abstract}
Summary
The concentrations of cortisol and testosterone (total and apparent free) in peripheral venous plasma were measured in 147 women. The results showed that there were no significant differences in concentrations between women with normal breasts, those with histologically confirmed benign or malignant breast tumours and those with cancer at other sites. There was, however, a significant increase with age in the concentrations of total $(P<0.005)$ and apparent free $(P<0.02)$ cortisol.
\end{abstract}

\section{Introduction}

The physiological role of androgenic steroids in women is obscure. The most potent androgen secreted by the ovary and adrenal cortex is testosterone, but it is present in peripheral venous plasma in much lower concentrations than the weaker androgens, dehydroepiandrosterone sulphate, dehydroepiandrosterone and androstenedione, which are mainly secreted by the adrenals. Previous studies have reported that abnormalities in androgen production and excretion are involved in the aetiology and clinical course of breast cancer (Bulbrook et al., 1962; Thomas et al., 1976). But investigation of testosterone metabolism in these patients has proved unrewarding (Jones, Ramsay and Collins, 1977b; Jones et al., 1977c).

It has been shown in women that breast tumour tissue is more active than other tissues in metabolizing testosterone to another active compound, $5 \alpha$-dihydrotestosterone ( $5 \alpha$-DHT) (Forchelli et al., 1967). The formation of $5 \alpha-D H T$ is greatest in the most undifferentiated cancers but does not occur in normal breast tissue (Jenkins and Ash, 1972). Thus, breast tumours are a target organ for circulating testosterone although the mechanism of action of this hormone is not understood (Wagner and Jung- 음 blut, 1976).

Many patients with breast cancer have impaired $\Phi$ glucose tolerance and obesity. Furthermore, the prevalence of diabetes mellitus is increased. The metabolic abnormalities may precede the development of clinical malignancy (Glicksman and Rav $ळ$ son, 1956), and it has been suggested that these. findings could be associated with an abnorm corticosteroid metabolism (Bell, Bulbrook and Desphande, 1967). Elevated levels of circulating corticosteroids have been reported in patients with breast cancer (Desphande, Hayward and Bulbrook, $\stackrel{\square}{\unrhd}$ 1965) and, as cortisol may suppress the cellular $\overrightarrow{\vec{P}}$ immune response in clinical and experimental situations (Fauci, 1976), it is possible that high levels of this steroid may facilitate tumour development and growth.

The results from earlier work have indicated an 3 association between abnormalities in androgen and corticosteroid metabolism in breast cancer (Bul- $-\frac{3}{3}$ brook et al., 1962; Bulbrook, Hayward and Spicer, §̊ 1971). Other investigators have suggested that the bulk of the circulating hormone which is strongly을 bound to plasma is biologically inert and only the $>$ unbound fraction is active (Rosenfield, 1971; Burke and Anderson, 1972). The present authors, therefore, $N$ measured the unbound concentrations of testosterone and cortisol in groups of women with breast $N$ disease and in various control groups in an attempt $\omega$ to clarify the role of these hormones in the aetiology of breast cancer.

\section{Patients and methods}

One hundred and forty-seven women between the $\frac{T}{0}$ ages of 24 and 90 years (mean 53 years) were studied. $\frac{}{\mathbb{D}}$ Forty-five of the women were hospital staff and $\frac{\widetilde{P}}{\mathbb{Q}}$ 
acted as normal controls. The remainder were classified in various groups: those with histologically confirmed benign $(n=20)$ or malignant breast tumours and those with non-metastic cancer at other sites $(n=11)$. (The malignant breast group was classified as early $(n=43)$ or advanced $(n=28)$ breast cancer according to the Manchester Classification (Wise, York Mason and Ackerman, 1971).) All gave their informed consent. The women were then categorized as being premenopausal, early postmenopausal (one to 10 years after the last menstrual period) and late postmenopausal (11 to 30 years after the last menstrual period). The postmenopausal groups were sub-divided as age-related changes in postmenopausal hormone concentrations have been demonstrated (Chakravarti et al., 1976).

Blood sampling was performed between 10.30 and 12.00 a.m., as there is evidence of a diurnal rhythm in the levels of circulating testosterone and cortisol (Tyler, Newton and Collins, 1975). Plasma was separated by centrifuging at $1000 \mathrm{rev} / \mathrm{min}$ for $15 \mathrm{~min}$ and immediately stored at $-20^{\circ} \mathrm{C}$. Sampling was carried out during a visit to the hospital between one and 2 years after surgery. The time of the menstrual cycle was not taken into consideration in women who still had menses, because the fluctuations in testosterone levels are small and the pattern variable (Tyler et al., 1975). All patients on drugs likely to affect the concentrations of testosterone and cortisol in plasma were excluded from study.

Plasma testosterone was measured in a liquid phase radio-immunoassay system comprising tritiated testosterone as labelled antigen, and an antiserum raised against testosterone-3-carboxy-methyloxime-bovine serum albumin. After equilibration, dextran-coated charcoal was used to separate the antibody bound fraction (Tyler et al., 1973). Under these conditions the cross-reaction with $5 \alpha$-DHT was less than $15 \%$ and the inter-assay variation was within $12 \%$.

Cortisol was determined by a similar liquid phase radioimmunoassay system comprising a tritiated antigen and antiserum raised in rabbits against cortisol-21-succinyl-bovine serum albumin.

Equilibrium dialysis was the technique chosen to separate the protein bound and unbound hormone. The apparatus used was similar to that previously described (Ellis and Elkins, 1975) and consisted of a stoppered Teflon cell which was divided into 2 compartments by visking tubing. An aliquot (one $\mathrm{ml}$ ) of the plasma sample was placed in the upper section and $5 \mathrm{ml}$ of hepes buffer ( $\mathrm{pH} \mathrm{7.4)}$ in the lower compartment. The cells were then incubated with continuous agitation at $37^{\circ} \mathrm{C}$ in a water bath.

Initially, experiments were carried out to ascertain the time necessary for dialysis. A plasma pool was collected from blood samples taken from normal menstruating women. A series of cells was then set up using aliquots of plasma from the pool. These $\stackrel{\varnothing}{\complement}$ were incubated over a 24-hr period with cells $C$ removed in duplicate at intervals. An $18-\mathrm{hr}$ period $\vec{F}$ of incubation was then chosen for subsequent studies.

For the estimations of cortisol the dialysate and plasma were extracted in ether and measured $\overrightarrow{\mathbb{D}}$ directly by radioimmunoassay. However, because of $\cong$ the smaller amounts of testosterone present in the os samples, it was necessary to measure these indirectly $\overrightarrow{0}$ by a radioactive tracer technique. After incubation $\overrightarrow{-}$ and extraction as before, the fractions were dried and $\vec{\omega}$ redissolved in buffer and the radioactivity counted. The total hormone concentration was estimated by radio-immunoassay and the amount of unbound hormone was then calculated using the following equation:

$$
\% \text { unbound }=\frac{\text { d.p.m. in dialysate } \times 100}{\text { d.p.m. added }}
$$

d.p.m. - disintegrations per minute.

\section{Evaluation}

The methods have been evaluated over a period of 17 months and the mean values for each parameter are shown in Tables 1 and 2.

The blank value refers to the result obtained fron the analysis of hormone-free plasma. The sensitivit is that value which is significantly different from the blank at the $95 \%$ confidence level. The bias is the mean recovery of known amounts of cortisol or testosterone to hormone-free plasma. The precision is the coefficient of variation on replicate analysis of a quality control sample.

TABLE 1. An evaluation of methods for the measurement of total and apparent free cortisol

\begin{tabular}{lcc}
\hline \multicolumn{1}{c}{ Parameter } & Total cortisol & Free cortisol \\
\hline Blank value (nmol/l) & $12 \cdot 4$ & $6 \cdot 3$ \\
Sensitivity (nmol/l) & 20.6 & $8 \cdot 9$ \\
Bias (\%) & -12 & - \\
Precision: & & \\
Intra-assay (\%) & 8.9 & $9 \cdot 4$ \\
Inter-assay (\%) & 21.6 & 23.6 \\
\hline
\end{tabular}

TABLE 2. An evaluation of methods for the measurement of total and apparent free testosterone

\begin{tabular}{lcc}
\hline \multicolumn{1}{c}{ Parameter } & Total testosterone Free testosterone \\
\hline Blank value (pmol/1) & 212 & - \\
Sensitivity (pmol/1) & 460 & 40 \\
Bias (\%) & $0 \cdot 1$ & - \\
Precision: & $3 \cdot 0$ & $7 \cdot 1$ \\
Intra-assay (\%) & $17 \cdot 3$ & $20 \cdot 4$ \\
Inter-assay (\%) & & \\
\hline
\end{tabular}




\section{Results}

The frequency with which the concentration of each hormone appeared was studied and it was concluded that the distribution was best described by the arithmetic mean and range.

\section{Testosterone}

The concentrations of apparent free and total plasma testosterone are shown in Figs. 1 and 2. An analysis of variance was performed and no significant differences were found between any of the clinical groups, whether premenopausal or postmenopausal, so far as apparent free $\left(F_{4}, 126=2.04\right)$ and total plasma testosterone $\left(F_{4}, 126=0.74\right)$ were concerned. No significant differences were found in apparent free $\left(F_{2}, 126=1.35\right)$ or total testosterone $\left(F_{2}\right.$, $126=0 \cdot 71)$ between the premenopausal and the 2 postmenopausal groups, irrespective of diagnosis.

\section{Cortisol}

The concentrations of apparent free and total plasma cortisol are shown in Figs. 3 and 4. An analysis of co-variance was performed, with age as the co-variate. No significant differences in apparent free $\left(F_{4}, 126=0.85\right)$ or total plasma cortisol $\left(F_{4}, 126=0.60\right)$ were found between any of the

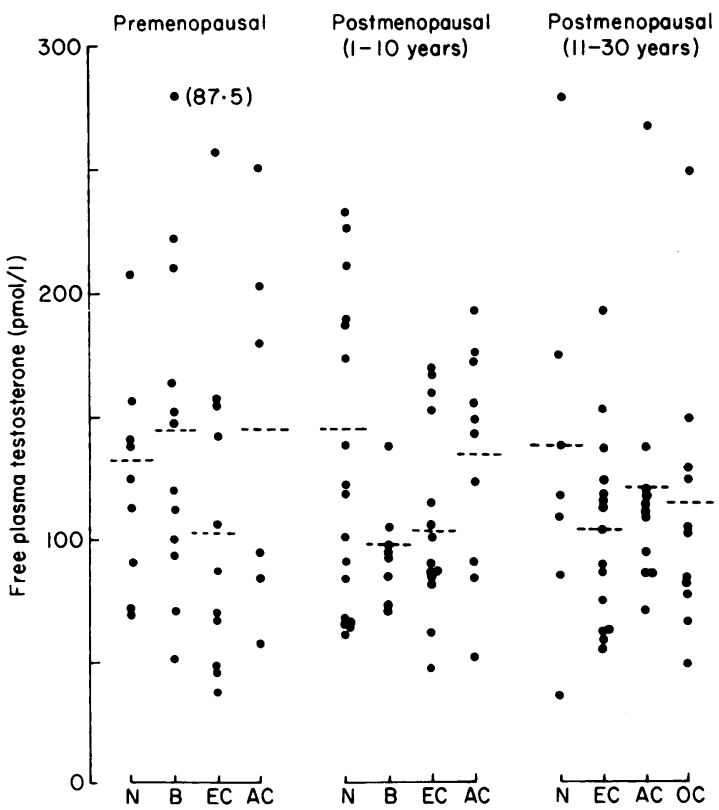

FIG. 1. The concentrations of apparent free plasma testosterone (pmol/1) in normal women $(\mathrm{N})$, women with benign breast disease (B), early and advanced breast cancer (EC; AC), and cancer at other sites (OC) are plotted. The groups are divided into premenopausal and 2 postmenopausal sub-groups. The means in each group are indicated by a dotted line.

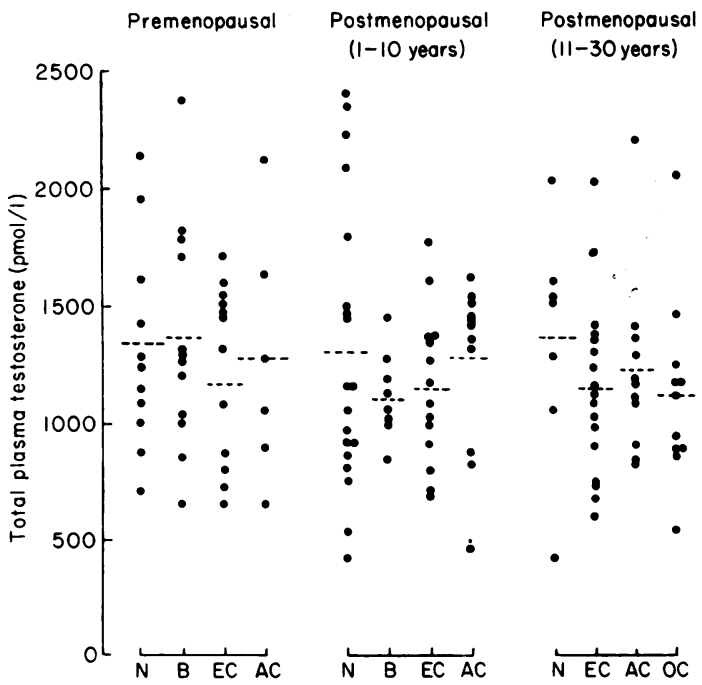

Fig. 2. The concentrations of total plasma testosterone (pmol/l) in normal women, women with benign breast disease, early and advanced breast cancer, and cancer at other sites are plotted (symbols as in Fig. 1). The groups are divided into premenopausal and 2 postmenopausal sub-groups. The means in each group are indicated by a dotted line.

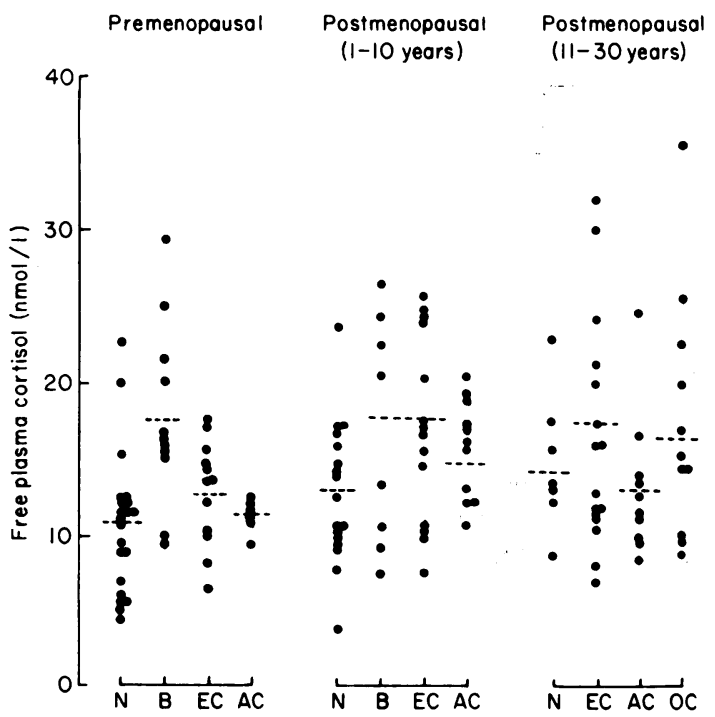

FIG. 3. The concentrations of apparent free plasma cortisol (nmol/l) in normal women, women with benign breast disease, early and advanced breast cancer, and cancer at other sites are plotted (symbols as in Fig. 1). The groups are divided into premenopausal and 2 postmenopausal sub-groups. The means in each group are indicated by a dotted line. 
clinical groups. No significant differences were found between the premenopausal and the 2 postmenopausal groups of apparent free $\left(F_{4}, 126=0.39\right)$ or total cortisol $\left(F_{2}, 126=0.39\right)$ using a similar analysis. However, when all the results were plotted together (Figs. 5 and 6) it was apparent that there was a linear relationship with age for both total $(m=0.68$ $\pm 0.24 ; \quad P<0.005)$ and apparent free cortisol $(m=0.34 \pm 0.14 ; P<0.02)$.

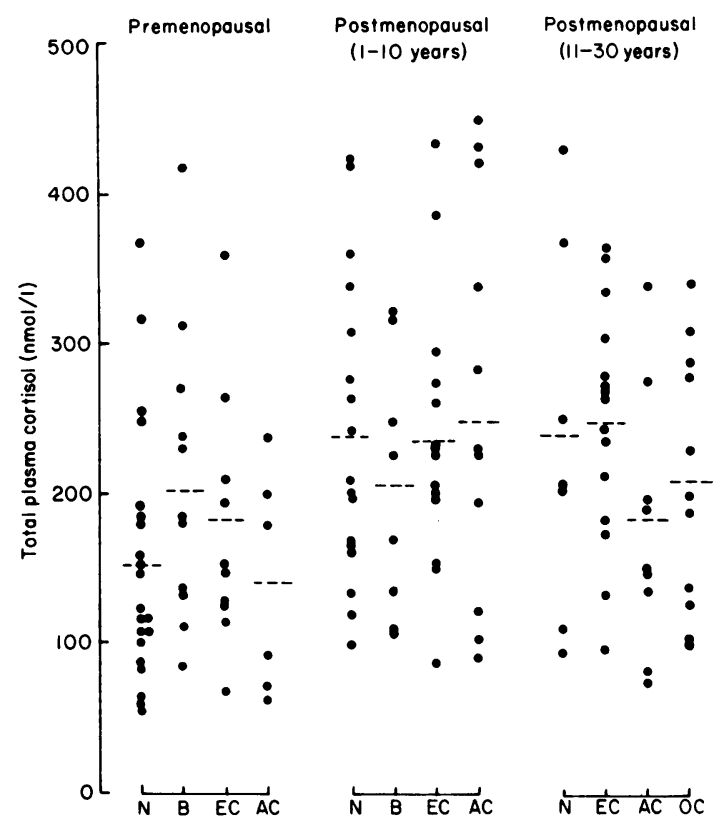

Fig. 4. The concentrations of total plasma cortisol (nmol/l) in normal women, women with benign breast disease, early and advanced breast cancer, and cancer at other sites are plotted (symbols as in Fig. 1). The groups are divided into premenopausal and 2 postmenopausal sub-groups. The means in each group are indicated by a dotted line.

\section{Discussion}

The concentrations of apparent free and total cortisol showed a statistically significant increase with age. The effect of age has previously been investigated in healthy subjects and total plasma cortisol was reported to be well maintained (Bliss et al., 1953). It is stated that the concentration of urinary metabolites of cortisol, i.e. 17-oxogenic steroids, decline in later life - the discrepancy between the plasma and urinary steroids being explained by a reduced rate of degradation of cortisol (West et al., 1961). The biological significance of the finding of a small increase in cortisol with advancing age is difficult to evaluate. The total and apparent free plasma testosterone concentrations were well maintained after the menopause and showed no decline with advancing age, which is in agreement with an earlier study (Chakravarti et al., 1976).

The concentrations of apparent free and total $c$ plasma testosterone were the same in plasma sampled from normal women, women with benign breast disease, early and advanced breast cancer, and cancer at other sites. Although total plasma testosterone is considered to be a good index of androgen status, several situations are known in which there is discordance between the plasma concentrations of the androgen and its clinical effects, for example, in pregnancy and hyperthyroidism. Circulating testosterone, however, is in dynamic equilibrium in human plasma with at least 3 forms of protein: (i) albumin; (ii) transcortin; (iii) sex hormonebinding globulin. The discrepancies mentioned between the clinical picture and the total plasma testosterone concentration are not so marked when apparent free plasma testosterone is used as the index of androgen function. The present findings are in agreement with previous studies from the same laboratory on the concentrations of plasma testosterone and its urinary metabolite, testosterone glucuronide (Jones et al., 1977 b. c). Thus to date, no abnormality has been found in the metabolism of testosterone in contradistinction to the wealth of evidence linking the production of dehydroepiandrog sterone sulphate, dehydroepiandrosterone and andros

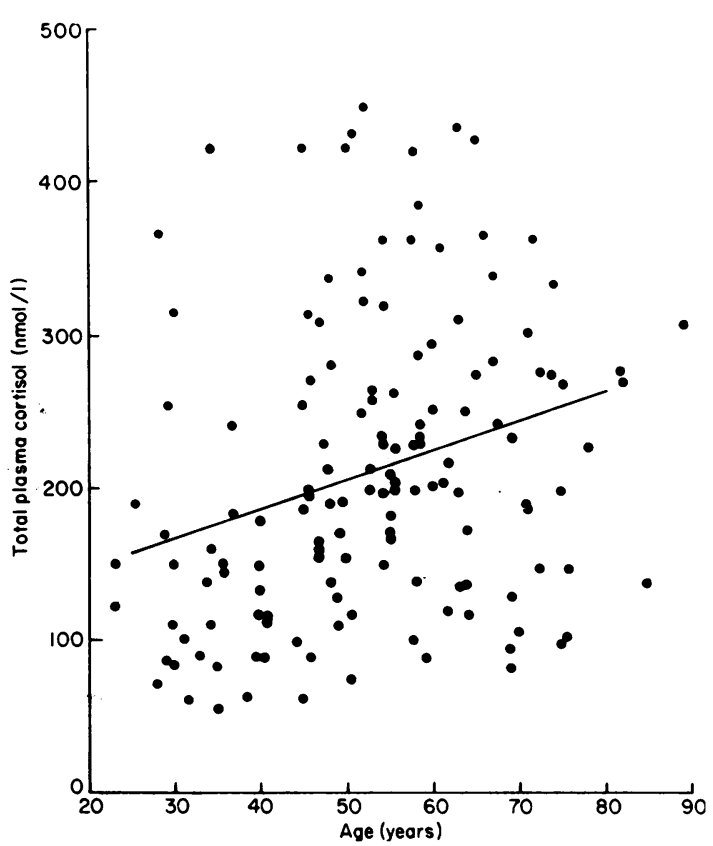

FIG. 5. The variation in the concentration of total plasma cortisol (nmol/1) is plotted against age in years. The regression line is shown. 
sterone with breast cancer (Bulbrook et al., 1962, 1971; Thomas et al., 1976).

There were no significant differences in the concentrations of apparent free and total plasma cortisol between the various groups studied. Circulating cortisol is in dynamic equilibrium with at least 2 groups of plasma protein: (i) albumin and (ii) transcortin. In pregnancy, the total concentration increases as the protein-bound fraction rises but the free concentration remains constant and there are no clinical signs of hyperandrenocorticism. The in vitro activity of cortisol correlates with the nonprotein bound concentration of the steroid and is probably the main factor determining the extent of cortisol action on cellular function. These findings would seem to be in agreement with a previous study (Bell et al., 1967) which found no significant difference in the binding activity of transcortin or in the 'mean per cent. bound'. However, the authors cannot support the conclusion that women with breast

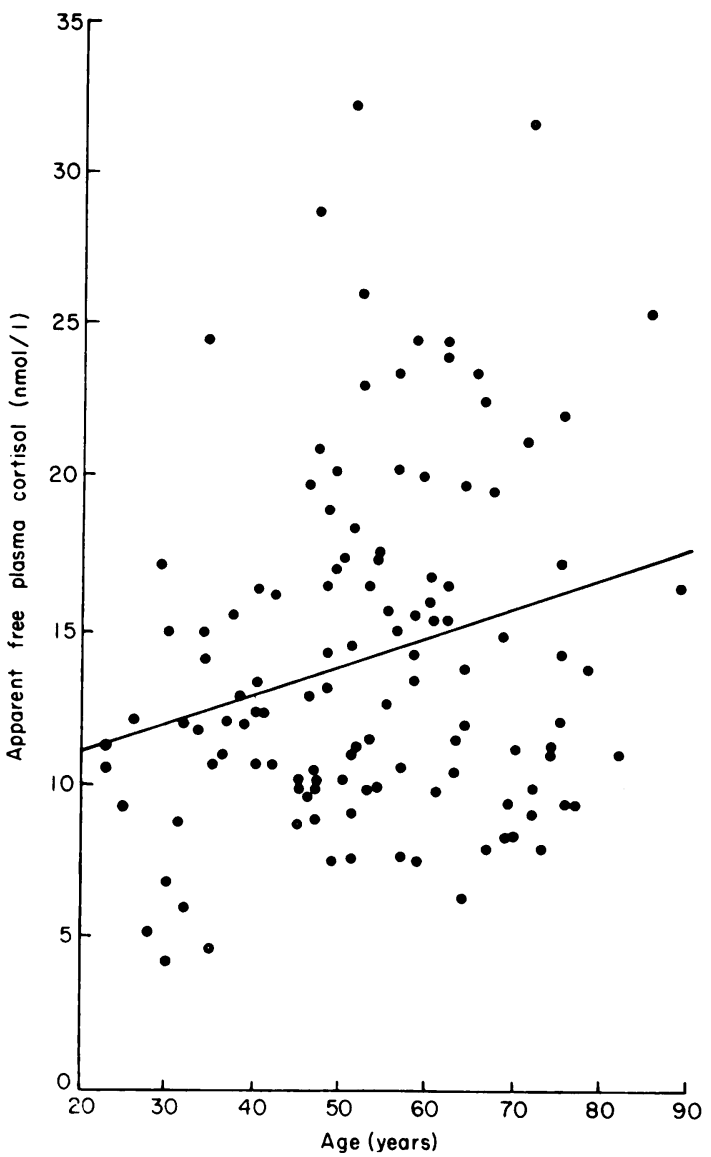

Fig. 6. The variation in the concentration of apparent free plasma cortisol (nmol/l) is plotted against age in years. The regression line is shown. cancer have more freely available cortisol; and their results differ from those of another group who found an increase in the carrier protein in the subjects studied (Sandberg, Slaunwhite and Carter, 1960).

Women with breast cancer have been variously reported as having abnormally high levels of plasma 17-hydroxycorticosteroids but normal urinary 17hydroxycorticosteroids (Desphande et al., 1965) or normal plasma and low urinary concentrations (Beck et al., 1966). These discrepancies cannot be explained by an abnormality in the protein bound component as suggested, and the authors have found no obvious difference in cortisol metabolism in these patients. The data agree with earlier studies measuring plasma cortisol (Jones et al., 1977a) and urinary free cortisol in similar groups of patients (Rose et al., 1977).

The epidemiological evidence that hormones are involved in the development of breast cancer is strong (MacMahon, Cole and Brown, 1973). However, studies on the plasma concentrations of hormones have generally not shown differences in those compounds known to be necessary for mammary growth and development (Swain, 1973; England et al., 1974; Jones et al., 1977a).

Free hormones can cross capillaries and reach cell receptors while protein-bound hormones cannot. For those hormones which circulate bound to a plasma protein it is best to equate hormone activity with apparent free (unbound) plasma hormone concentrations. If breast tissue contains cell receptors for the hormone concerned there is a shift of the free hormone from plasma across the capillaries to the interstitial fluid and then to the cell receptor. At a cellular level the dissociation rate of protein-bound hormone and the association rate of hormone uptake by the cell receptor are also important. The magnitude of the shift depends, of course, on the free hormone concentration in plasma, but also on the local blood flow, the rate of diffusion across the capillary wall, the concentration of cell receptors and the affinity of the cell receptor for the hormone concerned. We believe that it is in these areas that future studies on the effects of hormones in the aetiology of breast cancer should be directed.

\section{References}

Beck, J.C , Blair, A.J., Griffiths, M.M., Rosenfield, M.W. \& McGARRY, E.E. (1966) In search of hormonal factors as an aid in predicting the outcome of breast carcinoma. Proceedings of the Sixth Canadian Cancer Conference, pp. 3-34 (Ed by R. W. Begg). Oxford.

Bell, E., Bulbrook, R.D. \& Desphande, N. (1967) Transcortin in the plasma of patients with breast cancer. Lancet, ii, 395.

Bliss, E L . Sandberg, A.A., Nelson, D.H. \& Eik-Nes, K. (1953) The normal levels of 17-hydroxycorticosteroids in the peripheral blood of man. Journal of Clinical Investigation, 32, 818. 
Bulbrook, R.D., Hayward, J.L. \& SPICER, C.C. (1971) Relation between urinary androgen and corticoid excretion and subsequent breast cancer. Lancet, ii, 395.

Bulbrook, R.D., Hayward, J.L., Spicer, C.C. \& Thomas, B.S. (1962) A comparison between the urinary steroid excretion of normal women and women with advanced breast cancer. Lancet, ii, 1235.

BURKE, C.W. \& ANDERSON, D.C. (1972) Inter-relationship of unbound testosterone and oestradiol in human serum at $37^{\circ} \mathrm{C}$, and a biological role for sex hormone binding globulin. Journal of Endocrinology, 53, 26.

Chakravarti, S., Collins, W.P., Forecast, J.D., Newton, J.R., ORAM, D.W. \& STUdD, J.W.W. (1976) Hormonal profiles and the menopause. British Medical Journal. 2, 781.

Desphande, N., Hayward, J.L. \& Bulbrook, R.D. (1965) Plasma 17-hydroxycorticosteroids and 17-oxosteroids in patients with breast cancer and in normal women. Journal of Endocrinology, 32, 167.

Ellis, S.M. \& EKINS, R.P. (1975) The radioimmunoassay of serum free triiodothyronine and thyroxine. In: Radioimmunoassay and Clinical Biochemistry (Ed by C. A. Pasternak), pp. 187-194. Hayden, London.

England, P.C., Skinner, L.B., Cotterall, K.M. \& SellwOOD, R.A. (1974) Serum oestradiol-17ß in women with benign and malignant breast disease. British Journal of Cancer, 30, 571.

FAUCI, A.S. (1976) Glucosteroid therapy: mechanisms of action and clinical considerations. Annals of Internal Medicine, 84, 304.

Forchelli, E., Thomas, P.Z., Freyman, J.G., Parsons, D. \& Dorfman, R.I. (1967) Metabolism in vitro of ${ }^{4-14} \mathrm{C}$ testosterone in normal and malignant breast tissue. In: Current Concepts of Breast Cancer (Ed by A. Segaloff, K. K. Meyer and S. Debakey), pp. 99-108. Williams and Wilkins, Baltimore.

Glicksman, A.S. \& Rawson, R.W. (1956) Diabetes and altered carbohydrate metabolism in patients with cancer. Cancer, 9, 1127.

Jenkins, J.S. \& Ash, S. (1972) Metabolism of testosterone by human breast tumours. Lancet, ii, 513 .

Jones, M.K., Ramsay, I.D., Booth, M. \& Collins, W.P. (1977a) Hormone concentrations in postmenopausal patients with breast cancer. Clinical Oncology, 3, 177.

JoNeS, M.K., RAmSAY, I.D. \& Collins, W.P. (1977b) The concentrations of testosterone glucuronide in urine from women with breast tumours. British Journal of Cancer, 35, 885 .
Jones, M.K., Ramsay, I.D., Collins, W.P. \& DYer, G.I. (1977c) Plasma testosterone concentrations in patients with tumours of the breast. European Journal of Cancer, 13, 957.

MacMahon, B., Cole, P. \& Brown, J. (1973) Etiology of human breast cancer: a review. Journal of the National Cancer Institute, 50, 21.

Rose, D.P., Stauber, P., Thiel, A., Crowley, J.J. \& MilBRAITH, J.R. (1977) Plasma dehydroepiandrosterone sulphate, androstenedione and cortisol and urinary free cortisol excretion in breast cancer. European Journal of Cancer, 13, 43.

Rosenfield, R.L. (1971) Plasma testosterone-binding globulin and indexes of the concentrations of unbound plasma androgens in normal and hirsute subjects. Journal of Clinical Endocrinology and Metabolism, 32, 717.

Sandberg, A.A., Slaunwhite, W.R. \& Carter, A.C. (1960) Transcortin: a corticosteroid-binding protein of plasma. III. The effects of various steroids. Journal of Clinical Investigations, 39, 1914.

SwaIN, M C (1973) Plasma oestrogens and progesterone in benign and malignant breast disease. Acta endocrinologica. Copenhagen, 177 (Suppl.), 29.

Thomas, B.S., Kirkby, P., Symes, E.K. \& Wang, D.Y. (1976) Plasma dehydroepiandrosterone concentrations in normal women and in patients with benign and malignant breast disease. European Journal of Cancer, 12, 405.

Tyler, J.P.P., Hennam, J.R., Newton, J.R. \& Collins, W.P. (1973) Radioimmunoassay of plasma testosterone without chromatography: a comparison of four antisera, and the evolution of a novel approach to liquid scintillation counting. Steroids, 22, 871.

TyleR, J.P.P., Newton, J.R. \& Collins, W.P. (1975 Variations in the concentrations of testosterone in peri pheral venous plasma from healthy women. Acta endo crinologica. Copenhagen, 80, 542.

Wagner, R.K. \& Jungblut, P.W. (1976) Oestradiol and dihydrostestosterone receptors in normal and neoplastic human mammary tissue. Acta endocrinologica. Copenhagen, 82, 105.

West, C.D., Brown, H., Simons, E.L., Carter, D.B., Kumagai, L.F. \& EnLerT, E. (1961) Adrenal function and cortisol metabolism in old age. Clinical Research, 9, 102 (Abst).

Wise, L., York Mason, A. \& ACKerman, L.V. (1971) Local excision and irradiation: an alternative method for the treatment of early mammary cancer. Annals of Surgery, 174, 392. 